\title{
Deutschdidaktisches Wissen angehender Sekundarstufenlehrkräfte
}

\section{Testkonstruktion und Validierung}

\author{
Johannes König ${ }^{1}$ @ und Albert Bremerich-Vos² \\ ${ }^{1}$ Humanwissenschaftliche Fakultät, Universität zu Köln \\ ${ }^{2}$ Fakultät für Geisteswissenschaften, Universität Duisburg-Essen
}

\begin{abstract}
Zusammenfassung: Das fachdidaktische Wissen ist eine zentrale Facette professionellen Lehrerwissens. Im Gegensatz zu mathematischnaturwissenschaftlichen Fächern existieren bislang nur wenige fachdidaktische Wissenstests für sprachliche Fächer. Vorgestellt wird ein standardisierter Test zur Erfassung von fachdidaktischem Wissen angehender Deutschlehrkräfte der Sekundarstufen I und II, der im Rahmen des Projekts PlanvoLL-D entwickelt wurde. Datenbasis bilden die Testdaten von 378 Referendarinnen und Referendaren (Stichprobe 1) sowie 150 Lehramtsstudierenden (Stichprobe 2$)$ im Bachelor $(n=114)$ bzw. Master $(n=36)$. Zudem liegen für 85 dieser Studierenden eine Wiederholungstestung nach einem Jahr vor, für 27 Referendarinnen bzw. Referendare Befragungsdaten zur Unterrichtsqualität aus Sicht von 564 Schülerinnen und Schülern. Fragen zur Reliabilität des Tests an Stichprobe 1 und Stichprobe 2 sowie Fragen zur Kriteriumsvalidität werden geprüft. Die Ergebnisse zeigen, dass der Test eine reliable Messung an allen Stichproben erlaubt, die Retest-Stabilität ist hoch. Die Kriteriumsvalidität lässt sich hinsichtlich Übereinstimmungsvalidität, Methode der bekannten Gruppen und Vorhersagevalidität belegen.

Schlüsselwörter: Deutschdidaktik, Fachdidaktisches Wissen, Lehrerbildung, Kompetenz, Validierung
\end{abstract}

Future Secondary Teachers' German Pedagogical Content Knowledge: Test Construction and Validation

Abstract: Pedagogical content knowledge (PCK) is a core facet of professional teacher knowledge. In contrast to mathematics and science subjects, only few PCK tests exist for language subjects. This article presents a standardized test measuring the PCK of future teachers for German at secondary schools. The test was developed in the project PlanvoLL-D. Data were available from 378 teacher candidates (Sample 1) and 150 student teachers (Sample 2) during their bachelor $(n=114)$ or master studies $(n=36)$. Additionally, 85 of the student teachers were tested again 1 year later, and the instructional quality of 27 of the teacher candidates was rated by their students $(n=564)$ at school. Research questions concerning the reliability of the test applied to Sample 1 and Sample 2 as well as research questions regarding criterion validity were investigated. Findings show that the test allows for a reliable measurement for all samples, and retest stability is high. Criterion validity can be provided with regard to concurrent validity, known-groups method, and predictive validity.

Keywords: German Pedagogy, Pedagogical Content Knowledge, Teacher Education, Competence, Validation

Lehrpersonen benötigen für die erfolgreiche Bewältigung beruflicher Aufgaben berufsspezifisches Wissen (Bromme, 1992; Gitomer \& Zisk, 2015). In Bezug auf die Kernanforderung des Unterrichtens werden als zentrale Wissensfacetten Fachwissen, fachdidaktisches Wissen und pädagogisches Wissen unterschieden (Shulman, 1987; Baumert \& Kunter, 2006). Die Forschung zur Erfassung dieser Wissensfacetten verzeichnet in den vergangenen Jahren große Fortschritte (Baumert, 2016). Dies trifft insbesondere auf mathematisch-naturwissenschaftliche Fächer zu, während für sprachliche Fächer erst vereinzelt Ansätze existieren (König, Lammerding, Nold, Rohde, Strauß \& Tachtsoglou, 2016; Krauss, Lindl, Schilcher, Fricke, Göhring, Hofmann, Kirchhoff \& Mulder, 2017).
Im vorliegenden Beitrag wird zunächst ein standardisierter Test zur Erfassung von fachdidaktischem Wissen angehender Deutschlehrkräfte der Sekundarstufen vorgestellt, der im Rahmen des Projekts PlanvoLL-D (Die Bedeutung des professionellen Wissens angehender Deutschlehrkräfte für ihre Planung von Unterricht) entwickelt wurde, um mit einem Forschungsinstrument deutschdidaktisches Wissen als Outcome der universitären Lehrerausbildung zu erfassen. Dabei wurden Vorarbeiten aus dem Projekt TEDS-LT (Teacher Education and Development Study: Learning to Teach) genutzt, in dem studentische Kompetenzen ermittelt wurden. Der Test wurde angehenden Lehrpersonen aus den Bundesländern Berlin und Nordrhein-Westfalen zu Beginn ihres Referendariats vorgelegt ( $n=378$, Stichprobe 1), getestet wurden zudem 
Bachelor- und Master-Lehramtsstudierende der Universität zu Köln im jeweiligen 2. Fachsemester ( $n=150$, Stichprobe 2), gefolgt von einer Messwiederholung nach einem Jahr, an der sich 85 dieser Studierenden beteiligten. Für eine kleine Teilstichprobe der Referendarinnen und Referendare $(n=27)$ liegen zudem Befragungsdaten zur Unterrichtsqualität aus Sicht von $n=564$ unterrichteten Schülerinnen und Schülern vor. Auf Basis dieser Daten werden Analysen zur Reliabilität und Kriteriumsvalidität (Übereinstimmungsvalidität, Methode der bekannten Gruppen und Vorhersagevalidität) vorgestellt und im Anschluss diskutiert.

\section{Fachdidaktisches Wissen von Lehrkräften}

Das fachdidaktische Wissen (pedagogical content knowledge - PCK) gilt als zentrale kognitive Komponente der professionellen Kompetenz von Lehrkräften (Baumert \& Kunter, 2006). Sowohl theoretisch-konzeptionelle Arbeiten (im Überblick zuletzt Depaepe, Verschaffel \& Kelchtermans, 2013) als auch empirische Untersuchungen (Hill, Rowan \& Ball, 2005; Baumert et al., 2010) betonen die besondere Bedeutung des fachdidaktischen Wissens von Lehrkräften für die Steigerung von Fachleistungen ihrer Schülerinnen und Schüler (Blömeke, 2004; Lipowsky, 2006). Ohne fachdidaktisches Wissen, das die Lehrperson auf der kognitiven Ebene dazu befähigt, die „Sache“ in den pädagogischen Kontext des Lehrens und Lernens zu stellen (Shulman, 1986, 1987; Kennedy, 1990; Depaepe et al., 2013), ist Unterricht so gut wie nicht denkbar. Konsens besteht darin, dass mindestens die folgenden $\mathrm{Fa}$ cetten dieses Wissens zentral sind: das Wissen um Lehrmethoden, insbesondere die Konstruktion von Lern- und Übungsaufgaben, das Erklären von Fachinhalten, das Wissen über Lernstände und -prozesse einschließlich der Antizipation von Verständnisschwierigkeiten bzw. typischen Fehlern und Wissen im Zusammenhang mit der Beurteilung von fachspezifischen Leistungen. Lehrerinnen und Lehrer sind auf fachdidaktisches Wissen angewiesen, um ihrer pädagogischen Verantwortung den Lernenden gegenüber und der gesellschaftlichen Aufgabe von Schule gerecht zu werden (Kultusministerkonferenz [KMK], 2017).

Die empirische Erfassung von Professionswissen, speziell von fachdidaktischem Wissen als Element professioneller Kompetenz von angehenden wie berufstätigen Lehrpersonen, ist von grundlegender Bedeutung, etwa für die Arbeit an einer empirisch gestützten Theorie des Lehrerberufs und der Lehrerausbildung, für die vergleichende Evaluation der Wirksamkeit verschiedener Modelle der Lehrerbildung oder für die zukünftige Gestaltung von Karrierewegen im Lehrerberuf (Terhart, 2012; König et al.,
2018; Kaiser \& König, 2019). Für die Messung der Kompetenzen angehender Lehrerinnen und Lehrer sprechen auch globale Entwicklungen wie eine zunehmende Output-Steuerung in Bildungssystemen (KMK, 2017). So sind in den vergangenen Jahren zunehmend Ansätze zur Konzeptualisierung und Messung von fachdidaktischem Wissen für angehende und berufstätige Lehrkräfte entwickelt worden, wobei ein eindeutiger Schwerpunkt auf Mathematik als Unterrichtsfach liegt (Blömeke \& Delany, 2012; Callingham, Watson \& Burgess, 2012; Depaepe et al., 2013; Kleickmann et al., 2013). Entsprechende Arbeiten im Bereich der Lehrerforschung für das Fach Deutsch stehen dagegen noch sehr am Anfang. Diese sind von großer Relevanz, um in der Deutschdidaktik und der Germanistik solche Beiträge unter Rekurs auf empirische Befunde zu sichten und zu kommentieren, die sich auf die schon lange geführte Debatte beziehen, was Studierende, Referendarinnen und Referendare und Lehrkräfte wissen und können sollten (z. B. Gemeinsamer bildungspolitischer Arbeitskreis Germanistik und Deutschunterricht, 2009; Dehrmann \& Standke, 2012).

\section{Deutschdidaktische Forschung und die Testung von deutschdidaktischem Wissen}

2004 konstatierte Ingrid Kunze, dass Wissen und berufliche Kompetenzen von Deutschlehrkräften in der Deutschdidaktik noch kaum untersucht seien (Kunze, 2004, S. 191). Heutzutage stellt sich die Situation anders dar. Es erschienen seitdem z.B. Studien zum Wissen und Können von Lehrkräften im Kontext der Lehre von Lesestrategien (Bräuer, 2010), zu Präferenzen von Lehrkräften für Typen von Aufgaben im Literaturunterricht (Winkler, 2011), zu Orientierungen und Vorstellungen von Lehrkräften zur Leseförderung im Allgemeinen (Scherf, 2013), zum orthographischen Wissen von Lehrkräften und seinem Einfluss auf die Rechtschreibleistungen (Corvacho del Toro, 2013). Weitere Studien sind Referendarinnen und Referendaren gewidmet. So befragte z.B. Wieser (2008) 15 Berliner Lehramtsanwärterinnen und Lehramtsanwärter zu ihren literaturdidaktischen Konzepten. Ein kürzlich erschienener Sammelband mit überwiegend literaturdidaktischem Fokus hat den programmatischen Titel "Lehrende im Blick. Empirische Lehrerforschung in der Deutschdidaktik" (Bräuer \& Wieser, 2015). Bei den meisten dieser Arbeiten geht es nicht um Wissen im engeren Sinn und es handelt sich in der Regel um qualitative Studien mit geringen Zahlen Teilnehmender. Größere, quantitativ ausgerichtete Studien sind bislang nur TEDSLT (Bremerich-Vos \& Dämmer, 2013), ausschließlich auf Studierende ausgerichtet, und FALKO-D (Fachspezifische Lehrerkompetenzen - Deutsch; Pissarek \& Schilcher, 2017). 
Hier wurden sowohl Studierende, Referendarinnen bzw. Referendare als auch ausgebildete Lehrpersonen getestet (Krauss, Lindl, Schilcher \& Tepner, 2017, S. 31f.). Größere quantitativ ausgerichtete Studien sind nach wie vor rar.

In TEDS-LT wurden zu einem ersten Messzeitpunkt Lehramtsstudierende im 3. bis 5. Fachsemester im Hinblick auf ihr fachliches und fachdidaktisches Wissen getestet, später dann Studierende im 6. bis 8. Fachsemester. Das Fachwissen wurde unterteilt in literaturwissenschaftliches und linguistisches Wissen, beim fachdidaktischen Wissen gab es analog eine Einteilung in sprach- und literaturdidaktisches Wissen. Fachdidaktische Items bezogen sich z.B. auf die Kenntnis zentraler deutschdidaktischer Begriffe, auf die Einschätzung der Schwierigkeit von Aufgaben zum Lesen, auf die Beurteilung mündlicher Leistungen von Schülerinnen und Schülern, auf die Analyse von Rechtschreibfehlern und darauf, wie die Bestimmung von Satzgliedern gelehrt werden kann. Bei FALKO-D wurde insbesondere dem Präsentieren und Erklären und dem „Umgang mit Schülerkognitionen“ Rechnung getragen (Pissarek \& Schilcher, 2017, S. 78). Hinzu kam hier ein Aspekt unter dem Titel „Potential von Texten“, wobei folgende Annahme leitend war: „Je kompetenter eine Lehrkraft bei der Auswahl von Texten beziehungsweise bei der Beurteilung ihres Lernpotentials ist, desto besser ist sie in der Lage, einen zielorientierten und klar strukturierten Unterricht zu planen." (Pissarek \& Schilcher, 2017, S. 79). Bei FALKO-D kamen 13 Items zum Einsatz, die sich auf alle Lernbereiche des Deutschunterrichts beziehen, bei TEDS-LT waren es, nimmt man beide Messzeitpunkte zusammen, insgesamt 63 , mit denen ebenfalls alle Bereiche repräsentiert werden sollten (Bremerich-Vos \& Dämmer, 2013, S. 59).

Die Ergebnisse aus TEDS-LT zeigten, dass es über den Studienverlauf zu einem moderaten Zuwachs im deutschdidaktischen Wissen kam und Gymnasiallehramtsstudierende im Test geringfügig besser abschnitten als NichtGymnasiallehramtsstudierende. Mittelwertunterschiede zeigten sich auch in Abhängigkeit von den acht einbezogenen Studienorten, die Stichprobenrepräsentativität war in TEDS-LT allerdings deutlich eingeschränkt. In FALKO-D wurde je ein Test für das Fachwissen Deutsch und das deutschdidaktische Wissen entwickelt und bei einer Stichprobe von Lehramtsstudierenden der Universität Regensburg $(n=157)$ sowie einer Kombinationsstichprobe von Referendarinnen bzw. Referendaren und berufstätigen Lehrkräften $(n=114)$ eingesetzt. Diese Stichprobe schnitt in beiden Tests besser ab als jene, und innerhalb der Studierendenstichprobe schnitten Gymnasialstudierende besser ab als Nicht-Gymnasialstudierende.

Die Prüfung von Zusammenhängen zwischen fachdidaktischem und fachlichem Wissen wurde in beiden
Studien vorgenommen, bei TEDS-LT (Bremerich-Vos, Dämmer, Willenberg \& Schwippert, 2011, S. 62) zeigten sich zum ersten Messzeitpunkt latente Korrelationen des deutschdidaktischen Wissens mit dem literaturwissenschaftlichen $(\phi=.50)$ und dem linguistischen Wissen $(\phi=.52)$. In FALKO-D lag die latente Korrelation zwischen fachlichem und fachdidaktischem Wissen bei $\phi=.74$ (Pissarek \& Schilcher, 2017, S. 99). Eine darüber hinausgehende Prüfung des Zusammenhangs des deutschdidaktischen Wissens mit dem pädagogischen Wissen auf latenter Ebene erfolgte jedoch in keiner der beiden Studien. Folgt man Shulmans (1987) These, fachdidaktisches Wissen sei ein Amalgam aus fachlichem Wissen einerseits und pädagogischem Wissen andererseits, so wären höhere Korrelationen des fachdidaktischen Wissens mit fachlichem sowie pädagogischem Wissen zu erwarten als zwischen fachlichem und pädagogischem Wissen. Im sprachlichen Bereich liegen für das Fach Englisch als Fremdsprache erste Ergebnisse vor, die auf Basis einer Stichprobe von Referendarinnen und Referendaren ermittelt werden konnten und in diese Richtung weisen (König et al., 2016). Für das Fach Deutsch liegen unseres Wissens noch keine Ergebnisse aus der Prüfung der drei Wissensfacetten vor. Ferner liegen nach unserem Wissen keine Ergebnisse zur prognostischen Validität der in TEDS-LT bzw. FALKO-D entwickelten Tests vor, die z. B. Auskunft darüber geben, ob (angehende) Lehrpersonen mit umfangreicherem fachdidaktischen Wissen aus Sicht der von ihnen unterrichteten Schülerinnen und Schüler qualitätsvolleren Unterricht geben.

\section{Zur Testkonzeption im Projekt PlanvoLL-D}

Was die Papier-und-Bleistift-Testung des fachdidaktischen Wissens angeht, so erschien es sinnvoll, im Hinblick auf die curriculare Validität auf die von der Kultusministerkonferenz (KMK, 2008/2019) beschlossenen ländergemeinsamen inhaltlichen Anforderungen für die Fachwissenschaften und Fachdidaktiken in der Lehrerbildung, auf die länderübergreifenden Bildungsstandards im Fach Deutsch für den Mittleren Schulabschluss (KMK, 2004) und auf die Kerncurricula für das Fach Deutsch in den Schulformen der Sekundarstufe I in den beiden Bundesländern zurückzugreifen. Laut KMK haben sich die Lehrinhalte im Rahmen der Fachdidaktik Deutsch in der ersten Phase unter anderem auf Theorie und Konzepte des Deutschunterrichts und seiner Lernbereiche und auf Methoden und Verfahren der Textanalyse/Textinterpretation zu beziehen. In den Bildungsstandards, die den Kerncurricula in allen Bundesländern zugrunde liegen, werden die folgenden Lernbereiche unterschieden: Sprechen und Zuhören, Schreiben, Lesen - mit Texten und Medien umgehen so- 
wie Sprache und Sprachgebrauch untersuchen. Die Schülerinnen und Schüler sollen z.B. sach- und situationsangemessen mündlich argumentieren, argumentative Texte planen, formulieren und überarbeiten können, Grundregeln der Rechtschreibung beherrschen, Lesestrategien kennen und anwenden, im Umgang mit literarischen Texten analytische und produktive Methoden anwenden und Texte und Sätze im Hinblick auf ihre grammatischen Strukturen untersuchen können. Die didaktischen Lehrveranstaltungen in der ersten Phase sind thematisch auf die genannten Lernbereiche bezogen, wobei in der Regel zwischen sprach- und literaturdidaktischen Angeboten unterschieden wird. An den Universitäten in NRW z.B. müssen mindestens 15 Leistungspunkte in genuin fachdidaktischen Lehrveranstaltungen erworben werden. Es kommen Leistungspunkte für verschiedene schulische Praktika hinzu, vor allem für ein Praxissemester, das im Masterstudiengang angesiedelt ist. Es ist vorgeschrieben, dass bereits im Bachelorstudium fachdidaktische Lehre angeboten wird. Insofern ist davon auszugehen, dass Referendarinnen und Referendare mindestens fünf einschlägige Lehrveranstaltungen besucht haben, in der Regel mehr, z.B. Vorlesungen zur Einführung in die Fachdidaktik Deutsch und Seminare mit Titeln wie Schreibdidaktik, Lesedidaktik, Didaktik der Orthographie oder Didaktik der Grammatik. Auf der Basis der normativen Prämisse, dass die angehenden Lehrerinnen und Lehrer am Ende ihrer ersten Ausbildungsphase bzw. zu Beginn des Referendariats Kenntnisse in allen Lernbereichen des Deutschunterrichts haben sollten, wurden Items für jeden Bereich entwickelt (vgl. weiterführend den nachfolgenden Methodenteil). Mit PlanvoLL-D knüpfen wir in erster Linie an TEDS-LT an. Einige Items wurden in der ursprünglichen Version übernommen. Recht viele Items zum fachdidaktischen Wissen bei TEDS-LT betreffen allerdings nicht dessen Kernfacetten, sondern zielen allein auf begriffliches Wissen (z. B. auf eine Definition des Begriffs der Lesekompetenz). Auf solche Items wurde verzichtet, stattdessen wurden neue Items formuliert, die eine breitere Erfassung der Kernfacetten erlauben.

\section{Fragestellungen und Hypothesen}

Unsere erste Fragestellung bezieht sich auf die Reliabilität des neu entwickelten Tests zur Erfassung von deutschdidaktischem Wissen, während die übrigen Fragestellungen seine Kriteriumsvalidität betreffen, nämlich die Übereinstimmungsvalidität (Frage 2), die Methode der bekannten Gruppen (Frage 3) und die Vorhersagevalidität (Frage 4).

1. Lässt sich deutschdidaktisches Wissen von angehenden Lehrkräften reliabel messen? (a) Auf der Basis der normativen Prämisse, dass die von uns untersuchten angehenden Lehrkräfte während ihrer ersten Ausbildungsphase Kenntnisse in allen vom Test abgedeckten Bereichen des Deutschunterrichts erwerben sollten, erwarten wir eine reliable Messung deutschdidaktischen Wissens bei der Zielgruppe der Referendarinnen und Referendare. Wir erwarten auch eine reliable Messung bei einer Differenzierung dieser Zielgruppe nach Ausbildungsgängen. Darüber hinaus erwarten wir eine reliable Messung bei Lehramtsstudierenden in verschiedenen Stadien ihrer Ausbildung, d.h. im Bachelorsowie im Master-Studium.

(b) Bei wiederholter Anwendung des Tests nach einem Zeitintervall von einem Jahr interpretieren wir eine Korrelation der Messwerte zu den beiden Zeitpunkten als RetestStabilität. Dabei nehmen wir jedoch an, dass deutschdidaktisches Wissen innerhalb eines Studienjahrs Veränderungen unterliegt und somit keine hohe Stabilität besitzt. Im Gegensatz zu hohen oder sehr hohen Retest-Stabilitäten z. B. bei Intelligenztests $(r>.8$; Schmidt-Atzert \& Amelang, 2012, S. 139) nehmen wir eine nur moderate Höhe von $r>.5$ an.

2. Zeigen sich theoretisch zu erwartende Zusammenhänge mit anderen Facetten des professionellen Lehrerwissens sowie mit Indikatoren akademischer Leistungsfähigkeit?

(a) Deutschdidaktisches Wissen weist konzeptionell Überschneidungen mit dem Fachwissen sowie dem auf das Unterrichten bezogenen, pädagogischen Wissen auf. Aus theoretischer Sicht sollte deutschdidaktisches Wissen jedoch klar zu trennen sein vom fachlichen und pädagogischen Wissen (Shulman, 1987). Eine empirische Prüfung der Zusammenhänge sollte hohe positive Korrelationen des fachdidaktischen Wissens mit dem fachlichen und pädagogischen Wissen ergeben. Diese Korrelationen sollten statistisch signifikant höher sein als die Korrelation zwischen fachlichem und pädagogischem Wissen.

(b) Aufgrund der allgemein belegten prognostischen Validität der Abiturnote für Studienerfolg erwarten wir eine statistisch signifikante Korrelation der Abiturnote mit dem deutschdidaktischen Wissen. Da der Test deutschdidaktisches Wissen abbilden soll, das zumindest anteilig curricularer Gegenstand der universitären Lehrerausbildung ist, erwarten wir ferner eine statistisch signifikante Korrelation mit den Hochschulabschlussnoten.

3. Zeigen sich theoretisch zu erwartende Mittelwertunterschiede bei Subgruppen?

(a) Im Vergleich mit Lehramtsstudierenden im Bachelor- bzw. Master-Studium sollten angehende Lehrkräfte im Vorbereitungsdienst bzw. Referendariat, die bereits über einen Hochschulabschluss verfügen, durchschnittlich höhere Testleistungen erbringen. Denn angehende Lehrkräfte haben ihr fachdidaktisches Studium abge- 
schlossen und somit umfangreichere spezifische Lerngelegenheiten durchlaufen, die auf den Erwerb deutschdidaktischen Wissens zielten.

(b) Aufgrund einer stärkeren fachlichen Spezialisierung des Gymnasiallehramtes erwarten wir analog zu bisherigen Ergebnissen aus anderen Fächern (Mathematik, Englisch) und den Resultaten bisheriger deutschdidaktischer Testungen aus TEDS-LT und FALKO-D durchschnittlich höhere Testwerte der Referendarinnen und Referendare mit gymnasialer Ausrichtung.

4. Ist das deutschdidaktische Wissen ein Prädiktor für Unterrichtsqualität?

Im Sinne einer Vorhersagevalidität erwarten wir, dass das deutschdidaktische Wissen von Referendarinnen und Referendaren ein statistisch signifikanter Prädiktor für einzelne Merkmale der Unterrichtsqualität im Urteil der von ihnen unterrichteten Schülerinnen und Schüler ist. Schülerinnen und Schüler von Referendarinnen bzw. Referendaren mit umfangreicherem deutschdidaktischen Wissen sollten den Unterricht im Hinblick auf Aspekte der Basisdimensionen von Unterrichtsqualität (Klieme \& Rakoczy, 2008; Praetorius, Klieme, Herbert \& Pinger, 2018) auch positiver einschätzen. Dies sollte sich insbesondere in der kognitiven Aktivierung sowie in Merkmalen der konstruktiven Unterstützung manifestieren, da diese Merkmale eine größere fachliche Nähe aufweisen als zum Beispiel die Basisdimension der Klassenführung und bereits in Studien zum Mathematikunterricht durch fachdidaktisches Wissen vorhergesagt werden konnten (z. B. Baumert et al., 2010). Die Zusammenhänge sollten auch bestehen bleiben, wenn das pädagogische Wissen der Referendarinnen und Referendare kontrolliert wird.

\section{Methode}

\section{Stichproben}

Stichprobe 1: Sie besteht aus 378 angehenden Sekundarstufenlehrkräften für das Fach Deutsch aus den beiden Bundesländern Berlin und Nordrhein-Westfalen (NRW). Im Rahmen des Projekts PlanvoLL-D (König et al., 2017) ${ }^{1}$ wurden sie zu Beginn ihrer zweiten Ausbildungsphase (Vorbereitungsdienst bzw. Referendariat) im Frühjahr bzw.
Sommer 2016 befragt bzw. getestet. In beiden Bundesländern wurden zuerst die Ausbildungsinstitutionen (Zentren für schulpraktische Lehrerausbildung in NRW, Fachseminare in Berlin) gewählt (institutionelle Rücklaufquote $94 \%$ bzw. $72 \%)$, dann innerhalb dieser Einheiten alle angehenden Lehrkräfte der definierten Zielgruppe einbezogen (individuelle Rücklaufquote $68 \%$ bzw. 91\%). Die Datenerhebung konnte in den Ausbildungsinstitutionen durchgeführt werden, eine Teilnahme war jedoch nicht verpflichtend. Diese Erhebung vor Ort, bei der unter anderem die Tests zum deutschdidaktischen und pädagogischen Wissen administriert wurden, wurde von geschulten Projektmitarbeiterinnen durchgeführt und aus Zeitgründen im Rahmen eines Online-Surveys, bei welchem unter anderem der Test zum fachlichen Wissen administriert wurde, fortgesetzt (Teilnahmequote $70 \%$ ). Die angehenden Lehrkräfte aus NRW strebten das Lehramt für Haupt- / Real-/Gesamtschule (HRGe; $n=111$ ) bzw. für Gymnasium / Gesamtschule an (Gym/Ge; $n=178)$, jene aus Berlin das Kombinationslehramt für Integrierte Sekundarschulen / Gymnasium (ISS / Gym; $n=89$ ). Im Durchschnitt waren die angehenden Lehrkräfte 28.7 Jahre alt $(S D=4.5), 79.6 \%$ von ihnen waren weiblich. Eine Teilstichprobe der Referendarinnen und Referendare in NRW erklärte sich auf Anfrage bereit, eine Befragung der von ihnen unterrichteten Schülerinnen und Schüler zur Unterrichtsqualität durchzuführen. Somit liegt für 27 Referendarinnen bzw. Referendare und ihren Testwerten ein Datensatz von 27 Schulklassen mit 564 Schülerinnen und Schülern vor. Auf der Basis dieser Daten wird Fragestellung 4 bearbeitet. Trotz der sehr niedrigen Rücklaufquote mit $9 \%$ (27 von 289 angefragten Referendarinnen und Referendaren) konnte eine Selektivitätsanalyse keine signifikanten Mittelwerte mit praktischer Bedeutsamkeit in relevanten Untersuchungsvariablen aufzeigen - weder in den einzelnen Wissensvariablen noch in der Abitursowie den Hochschulnoten oder dem HISEI ${ }^{2}$ oder Alter (jeweils $p>.18, \eta^{2}<.01$ ). Auch fiel der jeweilige ChiQuadrat-Test auf überzufällige Verteilung von Teilnahme und Lehramt bzw. Geschlecht nicht signifikant aus (Lehramt: $p$ (2-seitig) = .148; Geschlecht: $p$ (2-seitig) = .084).

Stichprobe 2: Im Rahmen des Projekts ZuS (Zukunftsstrategie Lehrer*innenbildung Köln: Heterogenität und Inklusion gestalten) der Universität zu Köln wurde das deutschdidaktische Wissen von Lehramtsstudierenden mit dem-

\footnotetext{
Die vorliegende Arbeit entstand im Rahmen des Projekts „Die Bedeutung des professionellen Wissens angehender Deutschlehrkrafte für ihre Planung von Unterricht“" (PlanvoLL-D). PlanvoLL-D ist ein Verbundprojekt der Technischen Universität Berlin, der Universität Duisburg-Essen und der Universität zu Köln (Leitung: Prof. Dr. Albert Bremerich-Vos, Dr. Christiane Buchholtz, Prof. Dr. Johannes König), gefördert vom Bundesministerium für Bildung und Forschung (BMBF) im Rahmen der Linie „Kompetenzmodelle und Instrumente der Kompetenzerfassung im Hochschulsektor - Validierungen und methodische Innovationen" (KoKoHs). Förderkennzeichen: 01PK15014 A, 01PK15014B, 01PK15014C.

2 HISEl steht für Highest International Socio-Economic Index of Occupational Status, ein Maß des höchsten beruflichen Status in der Familie (Ganzeboom, De Graaf \& Treimann, 1992).
} 
selben Instrument getestet (König et al., 2018). ${ }^{3}$ Diese befanden sich im Sommersemester 2016 im 2. Fachsemester entweder ihres Bachelor- $(n=114)$ oder ihres Masterstudiums $(n=36)$. Ein Jahr später konnten $n=85$ dieser Studierenden erneut erreicht und getestet werden. Die Testdaten des ersten Messzeitpunktes von Stichprobe 2 von $n=150$ Lehramtsstudierenden verwenden wir für die Bearbeitung von Fragestellung 3a), die Messwiederholungsdaten der $n=85$ Panelisten nutzen wir für die Bearbeitung von Fragestellung 1b), d.h. für die Berechnung der RetestStabilität.

\section{Instrumente}

Der Test zur Erfassung des deutschdidaktischen Wissens wurde im Projekt PlanvoLL-D entwickelt, basierend auf Vorarbeiten aus TEDS-LT (Bremerich-Vos \& Dämmer, 2013). Es werden fünf Inhaltsbereiche unterschieden, die sich aus den Setzungen in den länderübergreifenden Bildungsstandards und den Kerncurricula für das Fach Deutsch ergeben: Sprechen und Zuhören, Schreiben, Rechtschreiben, Lesen und Sprache und Sprachgebrauch reflektieren. Im Bereich Sprechen und Zuhören wird z. B. Wissen zu zentralen Differenzen von Mündlichkeit und Schriftlichkeit erfragt, in der Domäne Schreiben geht es unter anderem um die Erhebung der Kenntnis von Methoden der Schreibförderung und von zentralen Merkmalen schulisch relevanter Textsorten bzw. -muster. Aufgaben zur Rechtschreibung zielen vor allem auf förderdiagnostisches Wissen, d.h. auf qualitative Analysen von Rechtschreibfehlern und darauf abgestimmte Förderstrategien. Aufgaben zum Kompetenzbereich Lesen haben mit Aspekten der als basal anzusehenden Leseflüssigkeit und mit Verfahren ihrer Förderung zu tun, darüber hinaus mit Lesestrategien und deren kontextsensibler Anwendung. In Lehrwerken gängige Aufgaben zu literarischen Texten sollen auf Modelle literarischen Lernens bezogen werden und es wird nach Wissen im Hinblick auf die Eignung und Anwendung von Methoden des handlungs- und produktionsorientierten Umgangs mit literarischen Texten gefragt. Darüber hinaus sollen Lernaufgaben zu literarischen Texten als mehr oder weniger schwierig eingestuft werden. Die Testaufgaben zur Sprachreflexion beziehen sich vor allem auf den klassischen Kern der Schulgrammatik, d.h. auf die Lehre von Wortarten und Satzgliedern und auf Proben bzw. Operationen zu ihrer Bestimmung.
In TEDS-LT waren einzelne dieser Inhaltsbereiche nicht durchgängig ihrem Gewicht in der ersten Phase der Ausbildung im Fach Deutsch entsprechend vertreten, d.h. leicht über- bzw. unterrepräsentiert. Deshalb wurden einzelne Testaufgaben, insbesondere solche mit geringerer Trennschärfe, ausgeschlossen und einige für PlanvoLL-D neu entwickelt. Insgesamt handelt es sich um 29 Testaufgaben, die sich auf die fünf Dimensionen verteilen (zwischen drei und elf Testaufgaben pro Dimension). Aus jeder Testaufgabe resultiert ein Testitem für die Skalierung. 13 Testaufgaben weisen ein single-choice-Format (eine richtige Antwortoption, ein Distraktor oder mehrere Distraktoren; s. die ersten drei Testaufgaben-Beispiele in Tabelle 1) auf, 16 Testaufgaben haben ein komplexes Format (constructed response; s. viertes und fünftes Testaufgaben-Beispiel in Tabelle 1). Hierfür waren zum Teil umfangreiche Kodierleitfäden zu entwickeln.

Als weitere Wissenstests zur Bearbeitung von Frage 2 verwendeten wir einen Test zur Erfassung des Fachwissens Deutsch sowie einen Test zur Erfassung des pädagogischen Wissens. Diese wurden in Stichprobe 1 eingesetzt. Das Fachwissen Deutsch ist durch die beiden akademischen Disziplinen Literaturwissenschaft und Linguistik geprägt. Der Test zum Fachwissen wurde im Projekt PlanvoLL-D wiederum auf Basis von Vorarbeiten aus TEDS-LT entwickelt und umfasst 48 Testaufgaben, je 24 für Fachwissen in Literaturwissenschaft und Linguistik (Tab. 1 und 2 im Elektronischen Supplement 1). Zur Erfassung des pädagogischen Wissens griffen wir auf den im Rahmen von TEDS-M entwickelten Test (König, Blömeke, Paine, Schmidt \& Hsieh, 2011) zurück, allerdings in einer Kurzfassung mit 40 Items (s. Testaufgaben-Beispiele in Tab. 3 im Elektronischen Supplement 1). Mit diesem Test aus TEDS-M werden unterrichtliche Herausforderungen, wie sie in der Forschung zur Unterrichtsqualität beschrieben werden, inhaltlich differenziert in die Inhaltsdimensionen Umgang mit Heterogenität, Strukturierung von Unterricht, Klassenführung, Motivierung und Leistungsbeurteilung. Jede der Dimensionen wird über mehrere komplexe Testaufgaben operationalisiert, die entsprechende unterrichtliche Herausforderungen zum Gegenstand haben.

Rund $20 \%$ aller Fragebögen von Stichprobe 1 wurden anhand der entwickelten Kodierleitfäden von zwei unabhängigen, geschulten Ratern doppelkodiert. Die Übereinstimmung wurde mit Cohen's Kappa berechnet. Sie liegt im Durchschnitt für die drei Tests zur Erfassung des Fachwissens, des fachdidaktischen und des pädagogischen Wissens in einem sehr guten Bereich sowohl für

\footnotetext{
3 Die Daten von Stichprobe 2 (Studierende) wurden im Rahmen des Handlungsfelds „Qualitätssicherung“ des Projekts „Zukunftsstrategie Lehrer*innenbildung Köln - Heterogenität und Inklusion gestalten“ (ZuS) der Universität zu Köln erhoben. ZuS wird im Rahmen der gemeinsamen „Qualitätsoffensive Lehrerbildung“von Bund und Ländern aus Mitteln des Bundesministeriums für Bildung und Forschung (BMBF) gefördert.
} 
Tabelle 1. Beispiel-Testaufgaben zur Erfassung von deutschdidaktischem Wissen

\begin{tabular}{|c|c|}
\hline Dimension & Beispiel-Testaufgabe \\
\hline Sprechen und Zuhören & 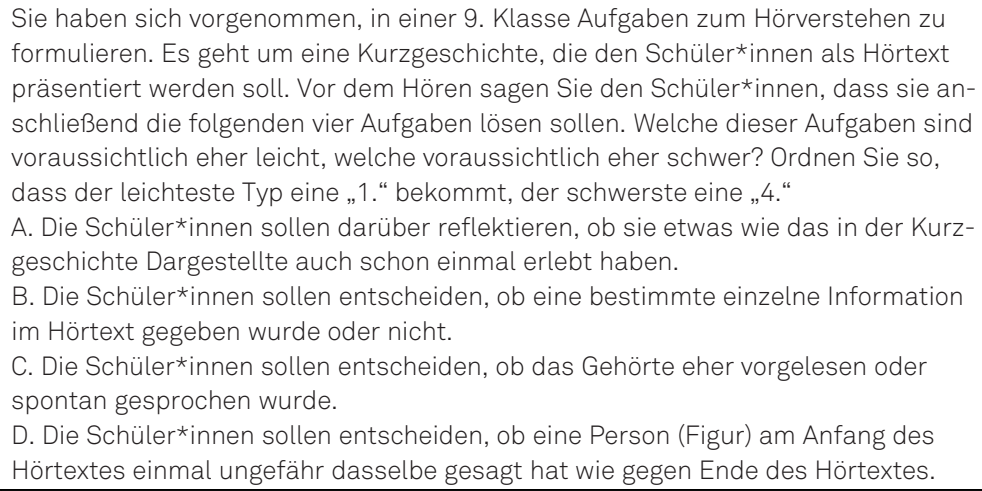 \\
\hline
\end{tabular}

Schreiben
In Lehrwerken für den Deutschunterricht in der Sekundarstufe wird oft zwischen
Bei welchen der folgenden Aufgaben wird eine lineare und bei welchen eine dia-
lektische Erörterung erwartet?
A. Warum sollten Jugendliche viel lesen?
B. Viele Hauptschülerlnnen bekommen keinen Ausbildungsplatz. Nenne einige Ur-
sachen.
C. Sollten Jugendliche einen eigenen Fernseher haben?
D. Zensuren für Lehrerlnnen! Setze Dich mit dieser Forderung auseinander.

Rechtschreiben Sie übernehmen eine 7. Klasse und wollen sich schnell über die Rechtschreibfähigkeiten der Schüler*innen informieren. Sie schreiben einen kleinen Test, in dem einzelne Wörter in Lücken und einige ganze Sätze zu schreiben sind. Es kommen u. a. die folgenden Wörter vor: Verkehrsschild, Tennisschläger, Bankräuber, Spinnennetz, Schiedsrichter. Thomas schreibt sie so: Verkehrsschilt, Tennischläger, Bankreuber, Spinnenetz, Schietsrichter. Sie wollen Thomas gezielt fördern, damit er solche Fehler nicht mehr macht. Was sollte Thomas in erster Linie lernen? A. Er sollte mehr auf die Lautung der Wörter achten.

B. Er sollte lernen, die Wörter in Silben zu zerlegen.

C. Er sollte sich jeweils die ganzen Wörter einprägen. D. Er sollte lernen, die Wörter in Morpheme zu zerlegen.

Lesen Lesen Sie bitte folgenden Text.

\section{Beste Geschichte meines Lebens}

Beste Geschichte meines Lebens. Anderthalb Maschinenseiten vielleicht. Autor vergessen; in der Zeitung gelesen. Zwei Schwerkranke im selben Zimmer. Einer an der Tür liegend, einer am Fenster. Nur der am Fenster kann hinaussehen. Der andere keinen größeren Wunsch, als das Fensterbett zu erhalten. Der am Fenster leidet darunter. Um den anderen zu entschädigen, erzählt er ihm stundenlang, was drauBen zu sehen ist, was draußen passiert. Eines Nachts bekommt er einen Erstickungsanfall. Der an der Tür könnte die Schwester rufen. Unterlässt es; denkt an das Bett. Am Morgen ist der andere tot; erstickt. Sein Fensterbett wird geräumt; der bisher an der Tür lag, erhält es. Sein Wunsch ist in Erfüllung gegangen. Gierig, erwartungsvoll wendet er das Gesicht zum Fenster. Nichts; nur eine Mauer. (Wolfdietrich Schnurre: Der Schattenfotograf. Aufzeichnungen. München. List 1978, 158)

Sie wollen diesen Text im Rahmen einer Reihe zu Kurzgeschichten in einer 9. Klasse einsetzen. Die Schüler*innen sollen eine handlungs- und produktionsorientierte Aufgabe bekommen. Deshalb präsentieren Sie den Text nicht ganz, sondern Sie lassen eine Stelle weg. Diese Leerstelle sollen die Schüler*innen dann so ausfüllen, wie sie es für sinnvoll halten.

Markieren Sie im Text die Stelle, die für das Streichen am ehesten geeignet ist.

Sprache und Sprachgebrauch reflektieren
Sie möchten Schüler*innen in einer 5. oder 6. Klasse erklären, warum das Adjektiv schnell ein typischeres Adjektiv ist als prima. Wie sollte Ihre Erklärung lauten?
Lösung/mögliche korrekte Antwort

Leichtester Typ (,,1.“): A.,

Schwerster Typ (,4.“): B.

Linear A. und B.; dialektisch

C. und D.

D. Wörter in Morpheme zerlegen
Nichts; nur eine Mauer sollte

unterstrichen werden. 
Tabelle 2. Kennwerte aus der Skalierung des deutschdidaktischen Wissenstests (Stichprobe 1 und 2 sowie Teilstichproben)

\begin{tabular}{|c|c|c|c|c|c|c|c|c|}
\hline Stichprobe & Messzeitpunkt & Gesamt- bzw. Teilstichprobe & $n$ & $\alpha$ & EAP-Reliabilität & Theta-Varianz & WMNSQ (min.-max.) & ID $(M)$ \\
\hline \multirow[t]{4}{*}{1} & & Gesamt & 378 & .62 & .65 & .35 & $.93-1.06$ & .31 \\
\hline & & HRGe (NRW) & 111 & .63 & .67 & .39 & $.95-1.11$ & .31 \\
\hline & & GymGe (NRW) & 178 & .64 & .67 & .38 & $.92-1.07$ & .33 \\
\hline & & ISS / Gym (Berlin) & 89 & .51 & .56 & .23 & $.91-1.11$ & .27 \\
\hline \multirow[t]{2}{*}{2} & 1 & Gesamt & 150 & .74 & .79 & .74 & $.92-1.11$ & .36 \\
\hline & 2 & Gesamt & 85 & .73 & .81 & .72 & $.87-1.16$ & .37 \\
\hline
\end{tabular}

Anmerkungen: WMNSQ = Weighted Mean Square, ID $(M)=$ Item-Discrimination (Mittelwert).

die in Berlin $(.87, .78, .91)$ als auch für die in NRW (.97, $.80, .89$ ) eingesetzten Bögen. Auch rund $20 \%$ der Bögen von Stichprobe 2 wurden auf entsprechende Weise doppelt kodiert und hier fällt die Übereinstimmung für die deutschdidaktischen Testfragen ebenfalls sehr gut aus $(\kappa>.75)$.

Zur Erfassung von Merkmalen der Unterrichtsqualität aus Schülersicht wurde eine Auswahl von Skalen aus der Third International Mathematics and Science Study (TIMSS; Waldis, Grob, Pauli \& Reusser, 2010) und dem Programme for International Student Assessment (PISA; Ramm et al., 2006) verwendet und sprachlich geringfügig modifiziert. In den Item-Formulierungen wurde insbesondere „Lehrer“ durch „Referendar“ ersetzt und mit einer Einleitungsfrage wurden die Schülerinnen und Schüler instruiert, die Items mit Bezug auf eine direkt zuvor gehaltene Lehrprobe der Referendarinnen bzw. Referendare zu beantworten.

Die Skalen erfassen die Basisdimensionen von Unterrichtsqualität: Kognitive Aktivierung (6 Items, z.B. „Unser Referendar stellt uns Fragen, über die wir wirklich nachdenken müssen.", $\alpha=.62$ ), Effektive Klassenführung (4 Items, z. B. „Unser Referendar weiß immer genau, was in der Klasse vor sich geht.", $\alpha=.67)$, Klarheit und Strukturiertheit (4 Items, z. B. „Unser Referendar hebt immer wieder hervor, was wichtig ist", $\alpha=.77$ ) und Unterstützung (4 Items, z. B. „Unser Referendar interessiert sich für den Lernfortschritt jedes einzelnen Schülers bzw. jeder einzelnen Schülerin.", $\alpha=.77$ ). Die Items liegen in Form von vierstufigen Likert-Skalen mit den Kategorien trifft nicht $z u$, trifft eher nicht $z u$, trifft eher $z u$, trifft $z u$ vor. Eine konfirmatorische Faktorenanalyse der vier Skalen als latente Variablen auf Schülerebene weist einen guten Modell-Fit auf $\left(\chi^{2}=270.994, d f=129, p<.001, \mathrm{CFI}=.932\right.$, RMSEA $=.044$, SRMR $=.044)$. Der jeweilige ICC für die vier Skalen variiert zwischen .124 (Kognitive Aktivierung) und .277 (Effektive Klassenführung), sodass Mehrebenenanalysen angebracht erscheinen (Julian, 2001).

\section{Ergebnisse}

\section{Skalenkennwerte und Reliabilität (Frage 1)}

Der deutschdidaktische Test wurde zunächst mithilfe der Software Conquest getrennt für jede Stichprobe IRT-skaliert. Tabelle 2 enthält zentrale Kennwerte. Insgesamt erweist sich der Test als reliabel und die Varianz der latenten Variable ist hinreichend. Item-Diskriminationswerte liegen mit durchschnittlich rund .3 in einem guten Bereich, der Weighted Mean Square (WMNSQ) jedes Items fällt in einen akzeptablen Bereich zwischen .8 und 1.20 (Bond \& Fox, 2007). Dies gilt für Stichprobe 1 und Stichprobe 2 wie auch für die Teilstichproben der drei Lehrämter in Stichprobe 1 und die beiden Messzeitpunkte in Stichprobe 2.

Um vergleichbare Fähigkeitswerte aus der IRT-Skalierung zu erhalten, die bei der Bearbeitung der nachfolgenden Fragestellungen nötig sind (z.B. Frage 1 zur Retest-Stabilität oder Frage 3), wurde wie folgt vorgegangen: Zugrunde gelegt wurden die EAP-Fähigkeitswerte aus der Gesamtskalierung von Stichprobe 1. Um vergleichbare Werte für Stichprobe $2 \mathrm{zu}$ erhalten, wurde die IRT-Skalierung für Stichprobe 2 erneut durchgeführt, wobei die Item-Parameter aus der Skalierung von Stichprobe $1 \mathrm{im}$ portiert und somit verankert wurden. Die Fähigkeitswerte aus Stichprobe 1 wurden anschließend für den Zweck einer besseren Lesbarkeit auf einen Mittelwert von 500 und eine Standardabweichung von 100 transformiert. Die Fähigkeitswerte aus Stichprobe 2 wurden unter Verwendung derselben Formel transformiert. Deskriptive Kennwerte zum durchschnittlichen Abschneiden enthält Tabelle 6. Auf dieser Basis wurde die Retest-Stabilität für die 85 Lehramtsstudierenden mit zweimaliger Messung berechnet. Diese ist mit $r=.64(p<.001)$ als moderat bis knapp hohe Korrelation zu interpretieren (Schmidt-Atzert \& Amelang, 2012, S. 139). 
Tabelle 3. Kennwerte der Item-Parcels des deutschdidaktischen Wissenstests $(n=378)$

\begin{tabular}{lccccc}
\hline & Sprechen und Zuhören & Schreiben & Rechtschreiben & Lesen & Sprache und Sprachgebrauch reflektieren \\
\hline Anzahl der Items & 3 & 4 & 5 & 11 & 6 \\
EAP-Reliabilitäta) & .40 & .43 & .50 & .57 & .57 \\
M & .39 & .50 & .46 & .45 & .48 \\
SD & .30 & .23 & .25 & .20 & .24 \\
SE & .02 & .01 & .01 & .01 & .01 \\
\hline
\end{tabular}

Anmerkung: a) Kennwerte entstammen einer mehrdimensionalen IRT-Skalierung mithilfe der Software Conquest.

\section{Beziehungen des deutschdidaktischen Wissens zu anderen Variablen (Frage 2)}

Zur Prüfung der Frage, inwieweit mit dem deutschdidaktischen Test eine eigenständige Facette des Professionswissens von Lehrkräften erfasst wird, wurden zwei weitere Tests einbezogen: ein Test zur Erfassung des Fachwissens Deutsch und ein Test zur Ermittlung des pädagogischen Wissens. Für beide Tests liegen ähnlich gute Kennwerte aus der IRT-Skalierung vor (Tab. 1 im Elektronischen Supplement 2). Im Fall des Fachwissens Deutsch zeigt sich jedoch, dass eine zweidimensionale Skalierung in die Dimensionen Literaturwissenschaft und Linguistik einer eindimensionalen Skalierung überlegen ist $\left(\Delta_{\text {Abweichung }}=\right.$ $106.49, \Delta_{\text {Parameter }}=2, p<.001$ ), sodass im Folgenden von einer Zweiteilung des Tests ausgegangen wird.

Für die weiteren Analysen wurden die Testitems, welche aus theoretischer Sicht eine Inhaltsdimension eines Tests abbilden, zu einer Subskala aufsummiert und als Indikatorvariable zur Prüfung der faktoriellen Struktur im Strukturgleichungsansatz mithilfe der Software Mplus (Version 7, Muthén \& Muthén, 2015) verwendet (Verfahren des sog. item parceling, Bandalos \& Finney, 2009). Dies ist im vorliegenden Fall insofern ratsam, als die nachfolgenden Modelle nur auf kleineren Teilstichproben basieren. Für den deutschdidaktischen Test ergaben sich somit fünf Indikatorvariablen (Tabelle 3).

Auch für die beiden weiteren Tests zum Fachwissen und pädagogischen Wissen wurden analog Item-Parcels gebildet. Im Falle des Fachwissenstests waren es je vier Indikatorvariablen für Literaturwissenschaft und Linguistik und auch für den Test zur Erfassung von pädagogischem Wissen wurden vier Indikatorvariablen gebildet (Tabellen 1 und 2 im Elektronischen Supplement 3). Um die Nachvollziehbarkeit der nachfolgenden Analysen zu gewährleisten, findet sich eine Dokumentation der Interkorrelationen aller Indikatorvariablen (Item-Parcels) der drei Tests in Tabelle 1 und Tabelle 2 im Elektronischen Supplement 4.

Zur Prüfung der Wissensstruktur spezifizierten wir ein Modell mit vier latenten Variablen, zwei zum Fachwissen Deutsch (Literaturwissenschaft und Linguistik), je eine zum deutschdidaktischen und pädagogischen Wissen (Modell 1). Dieses Modell wurde einem Modell gegenübergestellt, das lediglich eine latente Variable vorsieht, d.h. keine Differenzierung in Fachwissen Deutsch, deutschdidaktisches Wissen und pädagogisches Wissen vorsieht (Modell 2). Ferner wurden zwei Alternativmodelle mit jeweils zwei Faktoren spezifiziert: Im ersten bildet das deutschdidaktische Wissen zusammen mit dem pädagogischen Wissen einen Faktor, während das Fachwissen Deutsch den zweiten Faktor bildet (Modell 3). Im zweiten wird das deutschdidaktische Wissen mit dem Fachwissen Deutsch zu einem Faktor zusammengenommen und dem pädagogischen Wissen als zweiten Faktor gegenübergestellt (Modell 4). Während Modell 2 mit nur einer latenten Variable einen schlechten Fit aufweist und somit abgelehnt werden muss, ist der Fit des differenzierten Modells 1 mit vier latenten Variablen gut (s. Tabelle 4). Dieses differenzierte Modell ist auch jeweils den beiden weiteren zweidimensionalen Alternativmodellen überlegen (s. Tabelle 4), die deutschdidaktisches Wissen entweder mit dem pädagogischen (Modell 3) oder mit dem fachlichen Wissen faktoriell zusammenfassen (Modell 4).

Die Interkorrelationen der latenten Variablen des vierdimensionalen Modells sind in Tabelle 5 gelistet. Wie erwartet korreliert das deutschdidaktische Wissen sowohl mit dem pädagogischen Wissen als auch mit dem Fachwissen (Literaturwissenschaft, Linguistik) statistisch signifikant. Dagegen fallen die Zusammenhänge zwischen pädagogischem Wissen und Fachwissen statistisch signifikant niedriger aus als zwischen deutschdidaktischem Wissen und Fachwissen (Literaturwissenschaft: $z=4.401$, $p<.001$; Linguistik: $z=13.093, p<.001) .{ }^{4}$ Auffällig ist

\footnotetext{
Verwendet wurde der Signifikanztest nach Meng, Rosenthal und Rubin (1992), um die Unterschiede in der Höhe latenter Interkorrelationen zu prüfen.
} 
Tabelle 4. Ergebnisse aus Strukturanalysen zum Wissen (Modell-Fit)

\begin{tabular}{|c|c|c|c|c|c|c|c|}
\hline Modell & Dimensionen & $\chi^{2}$ & $d f$ & $p$ & $\mathrm{CFI}$ & RMSEA & SRMR \\
\hline 1 & $\begin{array}{l}\text { Vierdimensional: } \\
\text { FW1, FW2, FDW, PW }\end{array}$ & 176.79 & 113 & $<.001$ & .923 & .039 & .057 \\
\hline 2 & Eindimensional & 345.22 & 119 & $<.001$ & .728 & .071 & .085 \\
\hline 3 & $\begin{array}{l}\text { Zweidimensional: } \\
\text { FW1 \& FW2 (Faktor 1) vs. FDW \& PW (Faktor 2) }\end{array}$ & 234.29 & 118 & $<.001$ & .860 & .051 & .068 \\
\hline 4 & Zweidimensional: FW1, FW2 \& FDW (Faktor 1) vs. PW (Faktor 2) & 298.32 & 118 & $<.001$ & .783 & .064 & .079 \\
\hline
\end{tabular}

Anmerkungen: FW1 = Fachwissen Literaturwissenschaft, FW2 = Fachwissen Linguistik, FDW = Deutschdidaktisches Wissen, PW = Pädagogisches Wissen.

Tabelle 5. Interkorrelationen der latenten Variablen des vierdimensionalen Strukturmodells

(1) (2) (3)

\begin{tabular}{|c|c|c|c|c|}
\hline (1) & Fachwissen Literaturwissenschaft & & & \\
\hline (2) & Fachwissen Linguistik & $.709 * * *$ & & \\
\hline (3) & Deutschdidaktisches Wissen & $.254^{\star}$ & $.694 * \star \star$ & \\
\hline$(4)$ & Pädagogisches Wissen & .101 & $.317 * \star$ & $.764 * \star *$ \\
\hline
\end{tabular}

Anmerkungen: ${ }^{* * *} p<.001,{ }^{* *} p<.01,{ }^{*} p<.05$

dabei, dass das Fachwissen im Bereich Linguistik enger als das im Bereich Literaturwissenschaft mit dem pädagogischen Wissen $(z=5.653, p<.001)$ und mit dem deutschdidaktischen Wissen $(z=13.61, p<.001)$ zusammenhängt.

Zur Prüfung des deutschdidaktischen Wissens in Abgrenzung von allgemeinen kognitiven Fähigkeiten wurden Zusammenhänge mit Indikatoren akademischer Leistungsfähigkeit analysiert. Auf manifester Ebene korreliert das deutschdidaktische Wissen in Höhe von $r=-.19$ $(p<.001, n=372)$ negativ mit der Abiturnote, d.h. höhere Wissenstestwerte gehen mit einer besseren Abiturnote einher. Mit der Hochschulabschlussnote im Fach Deutsch korreliert das deutschdidaktische Wissen ebenfalls signifikant $(r=-.24, p<.001, n=255)$, ebenso mit der Note im weiteren Fach $(r=-.15, p<.05, n=248)$, lediglich die Korrelation mit der Note in den Bildungswissenschaften fällt schwächer aus. Hier wird das Signifikanzniveau von $5 \%$ knapp verfehlt $(r=-.12, p=.060, n=236)$.

\section{Methode der bekannten Gruppen (Frage 3)}

Ein weiterer Validierungsschritt erfolgte über die Methode der bekannten Gruppen a) nach Ausbildungsstadium und b) nach Schulform. Tabelle 6 enthält die Gruppenkennwerte. Zur Prüfung der Ausprägung des deutschdidaktischen Wissens in Abhängigkeit vom Stand der Ausbildung (jeweils 2. Fachsemester im Bachelor und Master, Eintritt ins Referendariat) wurden die Mittelwerte von
Stichprobe 1 (nur erster Messzeitpunkt) und von Bachelorbzw. Master-Studierenden aus Stichprobe 2 verglichen (Abbildung 1). Die drei Gruppen angehender Lehrkräfte unterscheiden sich statistisch signifikant $(F(2,525)=$ 78.701, $p<.001)$ mit mittlerer praktischer Bedeutsamkeit $\left(\eta^{2}=.231\right)$. Ergebnisse aus paarweisen Vergleichen unter Verwendung des Post-hoc-Scheffé-Tests zeigen, dass sich die Mittelwerte aller drei Gruppen signifikant voneinander unterscheiden (mindestens $p<.05$ ). Wie Abbildung $1 \mathrm{zu}$ entnehmen ist, haben Referendarinnen bzw. Referendare höhere Fähigkeitswerte als Master-Studierende und diese wiederum höhere Werte als BachelorStudierende (vgl. auch Tabelle 6). Ferner zeigt sich in der Längsschnittstichprobe von Lehramtsstudierenden, die an der Wiederholungsmessung ein Jahr später teilnahmen, ein statistisch signifikanter Zuwachs im deutschdidaktischen Wissen $(t(84)=-6.063, p<.001)$, der als von mittlerer praktischer Bedeutung anzusehen ist $(d=.56)$.

Zur Prüfung der Ausprägung des Wissens in Abhängigkeit von der Art der Ausbildung wurden die in Stichprobe 1 vertretenen drei Lehrämter HRGe und GymGe in NRW sowie ISS / Gym in Berlin hinsichtlich ihres durchschnittlichen Abschneidens verglichen. Sie unterscheiden sich statistisch signifikant $(F(2,375)=5.667, p<.01)$ und mit kleiner praktischer Bedeutsamkeit $\left(\eta^{2}=.029\right)$. Paarweise Vergleiche zeigen, dass sich sowohl GymGe als auch ISS / Gym vom Lehramt HRGe unterscheiden, während keine signifikanten Unterschiede zwischen GymGe und ISS / Gym bestehen. Wie den Mittelwerten in Tabelle 6 entnommen werden kann, schneiden die fachlich spe- 
600

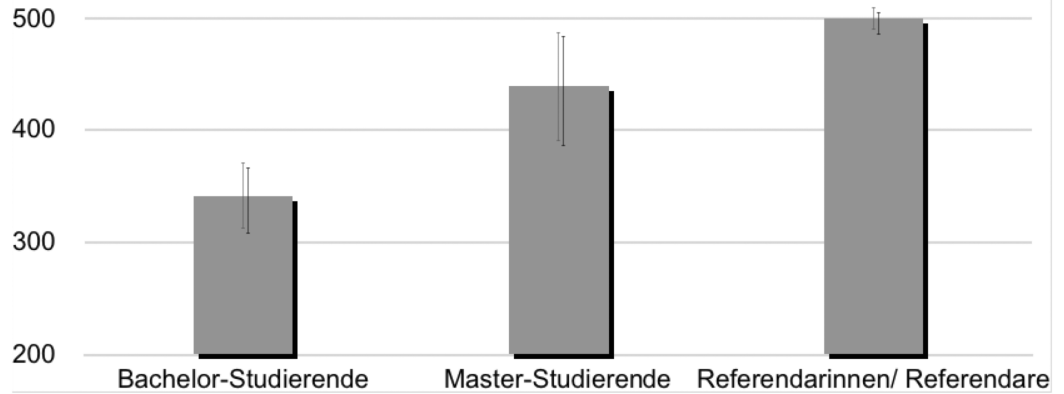

Abbildung 1. Mittlere Testleistung ( $95 \% \mathrm{KI})$ im deutschdidaktischen Wissen bei angehenden Sekundarstufenlehrkräften in unterschiedlichen Ausbildungsstadien ( $n=114$ Bachelor-Studierende: $M=341[312,370], n=36$ Master-Studierende: $M=439[390,488], n=378$ Referendarinnen/Referendare: $M=500[490,510])$

Tabelle 6. Deskriptive Statistiken zur erreichten Testleistung, gegliedert nach Stichprobe 1 und 2 sowie Teilstichproben

\begin{tabular}{|c|c|c|c|c|c|c|}
\hline Stichprobe & Messzeitpunkt & Gesamt- bzw. Teilstichprobe & $n$ & M & $S D$ & SE \\
\hline \multirow[t]{4}{*}{1} & & Gesamt & 378 & 500 & 100 & 5 \\
\hline & & HRGe (NRW) & 111 & 474 & 99 & 9 \\
\hline & & GymGe (NRW) & 178 & 508 & 103 & 8 \\
\hline & & ISS/Gym (Berlin) & 89 & 516 & 90 & 10 \\
\hline \multirow[t]{5}{*}{2} & 1 & Gesamt & 150 & 365 & 162 & 13 \\
\hline & & Bachelor (2. Fachsemester) & 114 & 341 & 160 & 15 \\
\hline & & Master (2. Fachsemester) & 36 & 439 & 146 & 25 \\
\hline & & Längsschnittprobanden & 85 & 391 & 143 & 16 \\
\hline & 2 & Gesamt & 85 & 471 & 146 & 16 \\
\hline
\end{tabular}

Anmerkung: Die Längsschnittprobanden setzen sich aus 55 Bachelor- und 30 Master-Studierenden zusammen.

zialisierten Lehrämter GymGe $(M=508)$ und ISS / Gym $(M=516)$ wie erwartet besser ab als das auf die Sekundarstufe I fokussierte Lehramt HRGe $(M=474)$.

\section{Vorhersagevalidität (Frage 4)}

Zur Prüfung der Vorhersagevalidität wurde für eine Teilstichprobe der Referendarinnen und Referendare aus NRW, für die auch Schülerdaten vorlagen, je ein Mehrebenenmodell für die einzelne Unterrichtsqualitätsskala berechnet (Tabelle 7). Datenbasis auf Individualebene waren die Daten von 564 Schülerinnen und Schülern aus 27 von den Referendarinnen bzw. Referendaren im Fach Deutsch der Sekundarstufe unterrichteten Schulklassen. Zunächst wurde jeweils in einem Kontrollmodell (M1, M4, M7, M10) ohne das deutschdidaktische Wissen die Varianzaufklärung der Kontrollvariablen Schulform, Alter und HISEI der Schülerinnen und Schüler geschätzt, wobei das Alter und der HISEI sowohl als Prädiktor auf Schülerebene (gruppenzentriert) als auch auf Klassenebene (als aggregiertes Merkmal) in die Regression eingingen.

Bei Einbezug des deutschdidaktischen Wissens (M2, M5, M8, M11) können auf Klassenebene gegenüber den Kon- trollmodellen zwischen 1\% (im Falle der Kognitiven Aktivierung) und $12 \%$ (im Falle der Klarheit und Strukturiertheit) der Varianz in den Urteilen der Schülerinnen und Schüler zur Unterrichtsqualität zusätzlich aufgeklärt werden. In allen Modellen ergeben sich positive Zusammenhänge zwischen deutschdidaktischem Wissen und Unterrichtsqualität. Im Fall der beiden Modelle zur Effektiven Klassenführung (M4) und zur Klarheit und Strukturiertheit (M6) erweist sich das deutschdidaktische Wissen als statistisch signifikanter Prädiktor $(\beta=.35, p<.05$, bzw. $\beta=.38, p<.05)$. Diese Ergebnisse bleiben auch bei Kontrolle des pädagogischen Wissens der Referendarinnen und Referendare bestehen (M3, M6, M9, M12).

\section{Diskussion}

Das Ziel der vorliegenden Untersuchung bestand darin, einen neu entwickelten Test zur Erfassung des deutschdidaktischen Wissens angehender Lehrkräfte in der Lehrerbildung als Forschungsinstrument zu präsentieren. Hierzu wurde die Konzeptualisierung vorgestellt und auf Basis von zwei Stichproben angehender Lehrkräfte zu Beginn 


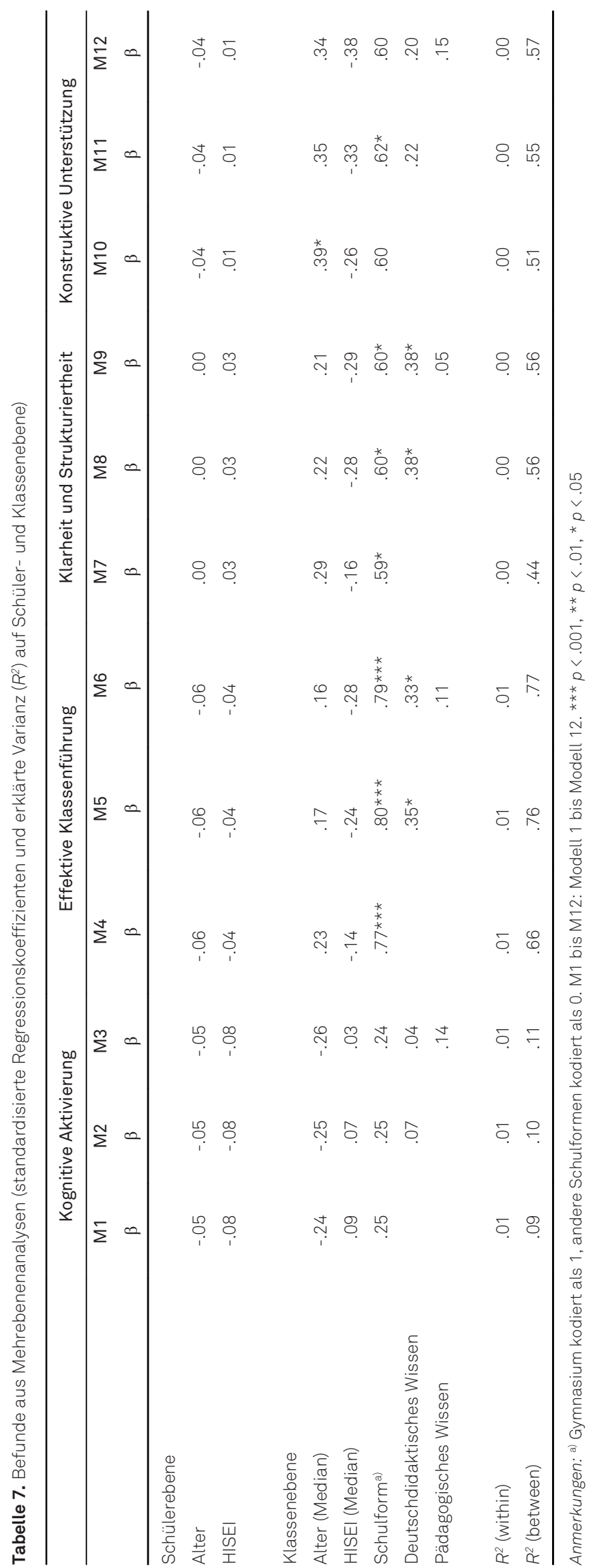


des Referendariats (Stichprobe 1) und im Studium (Stichprobe 2) die Reliabilität und Kriteriumsvalidität analysiert. Die Ergebnisse fallen weitgehend erwartungskonform aus.

\section{Reliabilität: Interne Konsistenz und Retest-Stabilität}

Der deutschdidaktische Wissenstest erwies sich als hinreichend reliabel und stabil. Sowohl an den beiden Stichproben 1 und 2 als auch an Teilstichproben (Stichprobe 1: Differenzierung nach Lehramt, Stichprobe 2: Messwiederholung) erlaubt der Test eine hinreichend reliable Messung. Die Retest-Stabilität kann als moderat bis knapp hoch interpretiert werden (Schmidt-Atzert \& Amelang, 2012, S. 139). Da es innerhalb des zugrunde gelegten Jahres zu deutlichen Veränderungen im deutschdidaktischen Wissen kommt (vgl. Tabelle 6), erachten wir die vorliegende Retest-Stabilität als hinreichend hoch für den Test.

\section{Validität}

Die Prüfung der Kriteriumsvalidität des Tests wurde in verschiedenen Schritten vorgenommen. Zunächst wurde die Übereinstimmungsvalidität analysiert, indem die Relationen des deutschdidaktischen Wissens und des fachlichen und pädagogischen Wissens analysiert wurden. Verglichen wurde ein Modell, in dem deutschdidaktisches Wissen von fachlichem bzw. pädagogischem Wissen nicht getrennt ist, mit einem differenzierten, mehrdimensionalen Modell mit diesen drei Facetten professionellen Wissens (Shulman, 1987), wobei fachliches Wissen aufgeteilt wurde in literaturwissenschaftliches und linguistisches, sodass es sich hierbei um ein vierdimensionales Modell handelt. Dieses vierdimensionale Modell erwies sich als überlegen, und zwar sowohl dem eindimensionalen Modell als auch zwei weiteren Alternativmodellen, in denen das fachdidaktische Wissen einmal dem fachlichen Wissen, ein andermal dem pädagogischen Wissen faktoriell zugeordnet wurde (Tabelle 4). Diese Befunde sprechen dafür, deutschdidaktisches Wissen als eigenen Bereich des Lehrerwissens zu definieren. Aus den Interkorrelationen der Wissensfacetten des vierdimensionalen Modells ergibt sich, dass deutschdidaktisches Wissen mit den beiden anderen Facetten nur teilweise gemeinsame Varianz aufweist (weniger als $60 \%$ mit dem pädagogischen Wissen, weniger als $50 \%$ mit linguistischem und weniger als $7 \%$ mit literaturwissenschaftlichem Wissen). Die Korrelationen sind allerdings statistisch signifikant höher als die zwischen fachlichem und pädagogischem Wissen. Dies lässt sich im Sinne der Hypothese von Shulman
(1987, S. 8) interpretieren, der zufolge fachdidaktisches Wissen als "special amalgam of content and pedagogy“ angesehen werden kann. Dass das deutschdidaktische Wissen mit linguistischem Wissen deutlich stärker als mit literaturwissenschaftlichem Wissen korreliert, ist ein Ergebnis unserer Analyse, das in zukünftigen Testungen des deutschdidaktischen Wissens bedacht werden sollte. So könnte z.B. der Anteil von Testaufgaben zum literaturdidaktischen Wissen im deutschdidaktischen Wissenstest erhöht werden. Unsere Analysen verstehen wir als Beitrag zur Diskussion um die Differenzierung des professionellen Lehrerwissens in die Kategorien des fachlichen, des fachdidaktischen und des pädagogischen Wissens (Shulman, 1987; Baumert \& Kunter, 2006). Die Tragfähigkeit dieser Differenzierung ist bislang für andere Domänen wie Mathematik (Blömeke, Busse, Kaiser, König \& Suhl, 2016) oder Fremdsprache Englisch (König et al., 2016) gezeigt worden. Die vorliegenden Analysen könnten zukünftig noch detaillierter fortgesetzt werden, etwa auf Ebene von Testaufgaben oder der für unsere Strukturanalysen verwendeten Item-Parcels. Hierzu sei auf die Tabellen aller Interkorrelationen von Item-Parcels verwiesen (Tabelle 1 und Tabelle 2 im Elektronischen Supplement 4).

Erwartungskonform korrelierte deutschdidaktisches Wissen sowohl mit der Abiturnote als auch mit den Hochschulabschlussnoten. Die Korrelation mit der Note in den Bildungswissenschaften fiel allerdings schwächer aus als jene mit den Noten in den Fächern. Möglicherweise kommt hier die Fokussierung auf fachliche Inhalte bei der Messung deutschdidaktischen Wissens zum Tragen.

Mithilfe der Methode bekannter Gruppen wurden Testleistungen von Bachelor- und Master-Studierenden mit den Leistungen der Referendarinnen und Referendare verglichen. Die Mittelwertvergleiche deuten darauf hin, dass mit fortgeschrittenem Ausbildungsstadium höhere Leistungen erbracht werden. Dies spricht für die curriculare Validität des deutschdidaktischen Tests. Es ist aber einschränkend $\mathrm{zu}$ berücksichtigen, dass es sich nicht um eine echte Längsschnittstichprobe handelt und dass Stichprobe 1 aus Referendarinnen und Referendaren aus zwei Bundesländern, Stichprobe 2 jedoch nur aus Studierenden einer Universität besteht. Der ersten Einschränkung konnte jedoch mit der Analyse von Messwiederholungsdaten zu Stichprobe 2 begegnet werden: Nach einem Jahr schnitten die erneut getesteten Studierenden im Test deutlich besser ab. Die Stichprobenmortalität war allerdings relativ groß (43\%). Der Zuwachs wird möglicherweise etwas überschätzt, denn es beteiligten sich Studierende, die bei der ersten Messung im Durchschnitt bereits eine etwas höhere Leistung $(M=391)$ erbracht hatten als die gesamte Stichprobe $2(M=365)$ (s. Tabelle 6). Dass 
bei dieser Analyse auf eine Imputation fehlender Werte verzichtet wurde und somit die Veränderungsmessung lediglich auf jenen Fällen beruht, die zu beiden Zeitpunkten auch an der Testung teilgenommen haben, muss als Limitation Berücksichtigung finden.

Ebenfalls mithilfe der Methode bekannter Gruppen wurde der Frage nachgegangen, ob Referendarinnen und Referendare in fachlich stärker spezialisierten gymnasialen Lehramtsstudiengängen im deutschdidaktischen Test besser abschneiden als Referendarinnen und Referendare, die sich allein auf das Unterrichten in der Sekundarstufe I vorbereiten. Hier zeigten sich statistisch signifikante Mittelwertunterschiede in der erwarteten Richtung. Der Befund entspricht Ergebnissen in anderen Domänen wie Mathematik (Blömeke, Kaiser, Döhrmann \& Lehmann, 2010; Krauss et al., 2008) und Englisch als Fremdsprache (König et al., 2016). Allerdings waren die Differenzen zugunsten der Gymnasialen nur von kleiner praktischer Bedeutsamkeit.

Erste Hinweise auf prognostische Validität erbrachte eine Mehrebenenanalyse. Es sollten Urteile von Schülerinnen und Schülern zu Facetten der Qualität des Unterrichts von 27 Referendarinnen und Referendaren aus Stichprobe 1 durch Rekurs auf deren fachdidaktisches Wissen erklärt werden. Das deutschdidaktische Wissen wurde mit verschiedenen Maßen zur Einschätzung der Unterrichtsqualität aus Schülersicht korreliert. Hypothesenkonform und in Übereinstimmung mit Befunden aus anderen Domänen wie der Mathematik (Baumert et al., 2010) ergaben sich positive Zusammenhänge mit den Merkmalen der Unterrichtsqualität. Allerdings fällt die durch das fachdidaktische Wissen der angehenden Lehrkräfte in der Qualitätsdimension kognitive Aktivierung aufgeklärte Varianz kaum ins Gewicht, hingegen waren die Merkmale der effektiven Klassenführung und der Klarheit und Strukturiertheit stärker durch deutschdidaktisches Wissen vorhersagbar, selbst bei Kontrolle pädagogischen Wissens. Möglicherweise hängt dies nicht zuletzt mit den Messeigenschaften der betreffenden Unterrichtsqualitätsskala zusammen. Denkbar ist aber auch, dass Unterrichtsmerkmale wie Klassenführung sowie Klarheit und Strukturiertheit, die für Referendarinnen und Referendare eine der größten Herausforderungen darstellen (z. B. Giallo \& Little, 2003), entsprechende kognitive Ressourcen - hier in Form ihres deutschdidaktischen Wissens - benötigen, sodass andere Merkmale wie die kognitive Aktivierung nur eingeschränkt zur Unterscheidung qualitätsvollen Unterrichts dienlich sind.

\section{Limitationen}

Als wesentliche Limitationen seien hervorgehoben: Obgleich Stichprobe 1 zufallsbasiert geplant und über ein aufwändiges zweistufiges Sampling-Verfahren gewonnen wurde, sind die kombinierten Rückläufe kritisch zu betrachten. Stichprobe 2 stammt von lediglich einer Universität. Bei den ergänzenden Stichprobendaten (Stichprobe 1: Teilstichprobe mit Schülerurteilen, Stichprobe 2: Wiederholungsmessung) können systematische (Selbst-)Ausleseprozesse bei der Datenerhebung nicht ausgeschlossen werden, wobei die berichtete Selektivitätsanalyse für die Teilstichprobe von Referendarinnen bzw. Referendaren mit Schülerurteilen hierzu allerdings keine Hinweise ergab. Es könnte jedoch auch sein, dass insbesondere Referendarinnen und Referendare in dieser Teilstichprobe vertreten sind, die insgesamt eher qualitätsvollen Unterricht halten und einer Erhebung von Schülerurteilen somit eher positiv gegenüberstanden. Generalisierungen der Befunde sind daher nur teilweise möglich. Das fachliche Wissen wurde online erhoben, sodass im Gegensatz zur Erhebung des deutschdidaktischen oder pädagogischen Wissens keine Testdurchführung unter Beobachtung stattfand. Dies hat möglicherweise Auswirkungen auf die Messqualität des Fachwissenstests. Hier wäre zukünftig ein Vergleich der online erhobenen Testdaten mit Testdaten, die unter Beobachtung gewonnen wurden, empfehlenswert. Die für Zusammenhangsanalysen verwendeten Noten wurden über Selbstberichte erhoben. Schließlich sei auch angemerkt, dass die Reliabilitäten des deutschdidaktischen Wissenstests je nach Stichprobe zwischen .62 und .74 variieren (Tabelle 1) und damit, insbesondere für die Referendarinnen und Referendare, nicht als sehr hoch einzustufen sind. Unter den verschiedenen Ursachen, die für die erreichte Reliabilität diskutiert werden können, sei hier auf die Herausforderung verwiesen, die sich mit einer eher allgemein gehaltenen Testung von deutschdidaktischem Wissen als inhaltlich relativ heterogener Wissensbereich verbindet. Zukünftige Testentwicklung könnte somit spezifische Teilbereiche deutschdidaktischen Wissens fokussieren und mit entsprechend höhere Anzahl von Testitems operationalisieren.

\section{Ausblick}

Der im Rahmen des Projekts PlanvoLL-D entwickelte Test zur Erfassung deutschdidaktischen Wissens ermöglicht insgesamt eine reliable und valide Messung in unterschiedlichen Ausbildungsstadien und bei unterschiedlichen Studiengängen, die für das Lehramt Deutsch der Sekundarstufe qualifizieren. Zwar lassen sich die hier erbrachten empirischen Befunde nur teilweise generali- 
sieren, sie sprechen jedoch dafür, den als Forschungsinstrument entwickelten deutschdidaktischen Wissenstest in weiterführenden Untersuchungen zum professionellen Wissen angehender Lehrkräfte einzusetzen. Insbesondere erscheinen weiterführende Zusammenhangsanalysen zu Lernergebnissen in Verbindung mit Ausbildungsgruppen und -stadien sowie Lerngelegenheiten sinnvoll. Ferner sollten die Validitätsprüfungen an der Zielgruppe berufstätiger Lehrkräfte fortgeführt werden, nicht zuletzt, um Einblicke in die prognostische Validität des deutschdidaktischen Wissens für Unterrichtsqualität und fachlichen Lernzuwachs bei Schülerinnen und Schülern zu erhalten.

\section{Elektronische Supplemente (ESM)}

Die elektronischen Supplemente sind mit der OnlineVersion dieses Artikels verfügbar unter https://doi.org/ 10.1026/0012-1924/a000251

ESM 1. Tabelle 1-3. Die Tabellen enthalten BeispielTestaufgaben der zusätzlich verwendeten Tests zur Erfassung von fachlichem und pädagogischem Wissen.

ESM 2. Tabelle 1. Die Tabelle 1 zeigt Kennwerte aus der IRT-Skalierung der zusätzlich verwendeten Tests zur Erfassung von fachlichem und pädagogischem Wissen.

ESM 3. Tabellen 1-2. Die Tabellen 1-2 zeigen die $\mathrm{Zu}$ sammenstellung der Items der zusätzlich verwendeten Tests zur Erfassung von fachlichem und pädagogischem Wissen zu Item-Parcels.

ESM 4. Tabelle 1. Die Tabelle 1 enthält die mithilfe von Mplus geschätzten Interkorrelationen aller Item-Parcels der drei verwendeten Tests zur Erfassung des Fachwissens Deutsch, des deutschdidaktischen Wissens und des pädagogischen Wissens.

\section{Literatur}

Bandalos, D. L. \& Finney, S. J. (2009). Item parceling issues in structural equation modeling. In G. A. Marcoulides \& R. E. Schumacker (Eds.), New Developments developments and Techniques techniques in Structural structural Equation equation Modeling modeling (pp. 269 -96). Mahwah, NJ: LEA.

Baumert, J. (2016). Leistungen, Leistungsfähigkeit und Leistungsgrenzen der empirischen Bildungsforschung: Das Beispiel von Large-Scale-Assessment-Studien zwischen Wissenschaft und Politik. Zeitschrift für Erziehungswissenschaft, 19 (Suppl. 1), 215-253. https://doi.org/10.1007/s11618-016-0704-4

Baumert, J. \& Kunter, M. (2006). Stichwort: Professionelle Kompetenz von Lehrkräften. Zeitschrift für Erziehungswissenschaft, 9, 469 - 520. https://doi.org/10.1007/s11618-006-0165-2

Baumert, J., Kunter, M., Blum, W., Brunner, M., Voss, T., Jordan, A. et al. (2010). Teachers' mathematical knowledge, cognitive ac- tivation in the classroom, and student progress. American Educational Research Journal, 47 (1), 133 - 180. https://doi.org/ 10.3102/0002831209345157

Blömeke, S. (2004). Empirische Befunde zur Wirksamkeit der Lehrerbildung. In S. Blömeke, P. Reinhold, G. Tulodziecki \& J. Wildt (Hrsg.), Handbuch Lehrerbildung (S. 59-91). Bad Heilbrunn: Klinkhardt.

Blömeke, S., Busse, A., Kaiser, G., König, J. \& Suhl, U. (2016). The relation between content-specific and general teacher knowledge and skills. Teaching and Teacher Education, 56, 35-46. https://doi.org/10.1016/j.tate.2016.02.003

Blömeke, S. \& Delaney, S. (2012). Assessment of teacher knowledge across countries: A review of the state of research. ZDM, 44, 223 - 247. https://doi.org/10.1007/978-94-007-6437-8_25

Blömeke, S., Kaiser, G., Döhrmann, M. \& Lehmann, R. (2010). Mathematisches und mathematikdidaktisches Wissen angehender Sekundarstufen-I-Lehrkräfte im internationalen Vergleich. In S. Blömeke, G. Kaiser \& R. Lehmann (Hrsg.), TEDS-M 2008 Professionelle Kompetenzen und Lerngelegenheiten angehender Mathematiklehrkräfte für die Sekundarstufe I im internationalen Vergleich (S. 197 -238). Münster: Waxmann.

Bond, T. \& Fox, C. (2007). Applying the Rasch model: Fundamental measurement in the human sciences (2nd ed.). Mahwah, $\mathrm{NJ}$ : Lawrence Erlbaum.

Bräuer, C. (2010). Könnerschaft und Kompetenz in der Leseausbildung. Theoretische und empirische Perspektiven. Weingarten \& München: Juventa.

Bräuer, C. \& Wieser, D. (2015). Lehrende im Blick. Empirische Lehrerforschung in der Deutschdidaktik. Wiesbaden: Springer.

Bremerich-Vos, A. \& Dämmer, J. (2013). Professionelles Wissen im Studienverlauf: Lehramt Deutsch. In S. Blömeke, A. BremerichVos, G. Kaiser, G. Nold, H. Haudeck, J.-U. Keßler \& K. Schwippert (Hrsg.), Professionelle Kompetenzen im Studienverlauf. Weitere Ergebnisse zur Deutsch-, Englisch- und Mathematiklehrerausbildung aus TEDS-LT (S. $47-75)$. Münster: Waxmann.

Bremerich-Vos, A., Dämmer, J., Willenberg, H. \& Schwippert, K. (2011). Professionelles Wissen von Studierenden des Lehramts Deutsch. In S. Blömeke, A. Bremerich- Vos, H. Haudeck, G. Kaiser, R. Lehmann, G. Nold, K. Schwippert \& H. Willenberg (Hrsg.) Kompetenzen von Lehramtsstudierenden in gering strukturierten Domänen. Erste Ergebnisse aus TEDS-LT (S. 47-76). Münster: Waxmann.

Bromme, R. (1992). Der Lehrer als Experte: Zur Psychologie des professionellen Wissens. Bern: Huber.

Callingham, R., Watson, J. \& Burgess, T. (2012). Uncertainty in mathematics education: What to do with statistics? In B. Perry, T. Lowrie, T. Logan, A. Macdonald \& J. Greenless (Eds.), Research in Mathematics Education in Australasia 2008-2011 (pp. 267-287). Leiden: Brill Sense.

Corvacho del Toro, I. (2013). Fachwissen von Grundschullehrkräften. Effekt auf die Rechtschreibleistung von Grundschülern. Bamberg: University of Bamberg Press.

Dehrmann, M. G. \& Standke, J. (2012). Germanistik und Lehrerbildung - Debatten und Positionen. Mitteilungen des Deutschen Germanistenverbandes, 59 (2)

Depaepe, F., Verschaffel, L. \& Kelchtermans, G. (2013). Pedagogical content knowledge: A systematic review of the way in which the concept has pervaded mathematics educational research. Teaching and Teacher Education, 34, $12-25$.

Ganzeboom, H. B., De Graaf, P. M. \& Treiman, D. J. (1992). A standard international socio-economic index of occupational status. Social Science Research, 21 (1), 1-56.

Gemeinsamer bildungspolitischer Arbeitskreis Germanistik und Deutschunterricht (2009). Ländergemeinsame inhaltliche Anforderungen für die Fachwissenschaften und Fachdidaktiken in der Lehrerinnen- und Lehrerbildung (Bamberger Empfeh- 
lungen). Didaktik Deutsch, 27, 91-107. http://www.didaktikdeutsch.de/article/27-berichte/

Giallo, R. \& Little, E. (2003). Classroom behavior problems: The relationship between preparedness, classroom experiences, and self-efficacy in graduate and student teachers. Australian Journal of Educational and Developmental Psychology, 3, 21 - 34.

Gitomer, D. H. \& Zisk, R. C. (2015). Knowing what teachers know. Review of Research in Education, 39, 1.

Hill, H. C., Rowan, B. \& Ball, D. L. (2005). Effects of teachers' mathematical knowledge for teaching on student achievement. American Educational Research Journal, 42, 371- 406.

Julian, M. W. (2001). The consequences of ignoring multilevel data structures in nonhierarchical covariance modeling. Structural Equation Modeling, 8, 325-352. https://doi.org/10.1207/ S15328007SEM0803_1

Kaiser, G. \& König, J. (2019). Competence measurement in (mathematics) teacher education and beyond: Implications for policy. Higher Education Policy, 32, 1-19. https://doi.org/10. 1057/s41307-019-00139-z

Kennedy, M. M. (1990). A survey of recent literature on teachers' subject matter knowledge (Vol. 90, No. 3). East Lansing, MI: National Center for Research on Teacher Education.

Kleickmann, T., Richter, D., Kunter, M., Elsner, J., Besser, M., Krauss, S. \& Baumert, J. (2013). Teachers' content knowledge and pedagogical content knowledge: The role of structural differences in teacher education. Journal of Teacher Education, 64 (1), 90 - 106. https://doi.org/10.1177\%2F0022487112460398

Klieme, E. \& Rakoczy, K. (2008). Empirische Unterrichtsforschung und Fachdidaktik. Outcome-orientierte Messung und Prozessqualität des Unterrichts. Zeitschrift für Pädagogik, 54, 222 - 237.

König, J. (2018). Erziehungswissenschaft und der Erwerb professioneller Kompetenz angehender Lehrkräfte. In J. Böhme, C. Cramer \& C. Bressler (Hrsg.), Erziehungswissenschaft und Lehrerbildung im Widerstreit (S. 62 -81). Bad Heilbrunn: Klinkhardt.

König, J., Blömeke, S., Paine, L., Schmidt, B. \& Hsieh, F-J. (2011). General pedagogical knowledge of future middle school teachers. On the complex ecology of teacher education in the United States, Germany, and Taiwan. Journal of Teacher Education, 62, 188 - 201. https://doi.org/10.1177\%2F0022487110388664

König, J., Bremerich-Vos, A., Buchholtz, C., Lammerding, S., Strauß, S., Fladung, I. et al. (2017). Die Bedeutung des Professionswissens von Referendarinnen und Referendaren mit Fach Deutsch für ihre Planungskompetenz (PlanvoLL-D). In S. Wernke \& K. Zierer (Hrsg.), Die Unterrichtsplanung: Ein in Vergessenheit geratener Kompetenzbereich?! Status Quo und Perspektiven aus Sicht der empirischen Forschung (S. 121-133). Bad Heilbrunn: Klinkhardt.

König, J., Doll, J., Buchholtz, N., Förster, S., Kaspar, K., Rühl, A.-M. et al. (2018). Pädagogisches Wissen versus fachdidaktisches Wissen? Struktur des professionellen Wissens bei angehenden Deutsch-, Englisch- und Mathematiklehrkräften im Studium. Zeitschrift für Erziehungswissenschaft, 21, 611-648. https://doi. org/10.1007/s11618-017-0765-z

König, J., Lammerding, S., Nold, G., Rohde, A., Strauß, S. \& Tachtsoglou, S. (2016). Teachers' professional knowledge for teaching English as a foreign language: Assessing the outcomes of teacher education. Journal of Teacher Education, 67, 320 - 337. https://doi.org/10.1177\%2F0022487116644956

Krauss, S., Brunner, M., Kunter, M., Baumert, J., Blum, W., Neubrand, M. et al. (2008). Pedagogical content knowledge and content knowledge of secondary mathematics teachers. Journal of Educational Psychology, 100, 716-725.

Krauss, S., Lindl, A., Schilcher, A., Fricke, M., Göhring, A., Hofmann, A., Kirchhoff, P. \& Mulder, R. H. (Hrsg.) (2017). FALKO: Fachspezifische Lehrerkompetenzen. Münster: Waxmann.
Krauss, S., Lindl, A., Schilcher, A. \& Tepner, O. (2017). Das Forschungsprojekt FALKO - ein einleitender Überblick. In S. Krauss, A. Lindl, A. Schilcher, M. Fricke, A. Göhring, B. Hofmann, P. Kirchhoff \& R. H. Mulder (Hrsg.), FALKO: Fachspezifische Lehrerkompetenzen (S. 9-65). Münster: Waxmann.

Kultusministerkonferenz (2004). Bildungsstandards im Fach Deutsch für den Mittleren Schulabschluss - Beschluss vom 15. 10.2003. München: Luchterhand.

Kultusministerkonferenz (2008/2019). Ländergemeinsame inhaltliche Anforderungen für die Fachwissenschaften und Fachdidaktiken in der Lehrerbildung. (Beschluss der Kultusministerkonferenz vom 16.10. 2008 i.d. F. Vom 16.05.2019)

Kultusministerkonferenz (2017). Ländergemeinsame inhaltliche Anforderungen für die Fachwissenschaften und Fachdidaktiken in der Lehrerbildung (Beschluss der KMK vom 16.10.2008 in der Fassung vom 12.10.2017) [Zugriff unter 2008_10_16Fachprofile-Lehrerbildung.pdf]

Kunze. I. (2004). Konzepte von Deutschunterricht. Eine Studie zu individuellen didaktischen Theorien von Lehrerinnen und Lehrern. Wiesbaden: VS Verlag für Sozialwissenschaften.

Lipowsky, F. (2006). Auf den Lehrer kommt es an. Empirische Evidenzen für Zusammenhänge zwischen Lehrerkompetenzen, Lehrerhandeln und dem Lernen der Schüler (Zeitschrift für Pädagogik: 51. Beiheft, S. 47-70). Weinheim: Beltz.

Meng, X.-L., Rosenthal, R. \& Rubin, D. B. (1992). Comparing correlated correlation coefficients. Psychological Bulletin, 111, 172175.

Muthén, L. K. \& Muthén, B. O. (2015). Mplus user's guide (7th ed.). Los Angeles, CA: Muthén \& Muthén.

Pissarek, M. \& Schilcher, A. (2017). FALKO-D: Die Untersuchung des Professionswissens von Deutschlehrenden. In S. Krauss, A. Lindl, A. Schilcher, M. Fricke, A. Göhring, B. Hofmann, P. Kirchhoff \& R. H. Mulder (Hrsg.), FALKO: Fachspezifische Lehrerkompetenzen (S. 67-111). Münster: Waxmann.

Praetorius, A. K., Klieme, E., Herbert, B. \& Pinger, P. (2018). Generic dimensions of teaching quality: The German framework of Three Basic Dimensions. ZDM Mathematics Education, 50, 407 - 426. https://doi.org/10.1007/s11858-018-0918-4

Ramm, G., Prenzel, M., Baumert, J., Blum, W., Lehmann, R., Leutner, D. et al. (2006). PISA 2003: Dokumentation der Erhebungsinstrumente. Münster: Waxmann.

Scherf, D. (2013). Leseförderung aus Lehrersicht. Eine qualitativeempirische Untersuchung professionellen Wissens. Wiesbaden: Springer.

Schmidt-Atzert, L. \& Amelang, M. (2012). Psychologische Diagnostik. Berlin, Heidelberg: Springer.

Shulman, L. S. (1986). Those who understand: Knowledge growth in teaching. Educational Researcher, 15 (2), 4-14. https://doi. org/10.2307/1175860

Shulman, L. S. (1987). Knowledge and teaching: Foundations of the new reform. Harvard Educational Review, 57, 1-23. https:// doi.org/10.17763/haer.57.1.j463w79r56455411

Terhart, E. (2012). Wie wirkt Lehrerbildung? Forschungsprobleme und Gestaltungsfragen. Zeitschrift für Bildungsforschung, 2 (1), 3-21. https://doi.org/10.1007/s35834-012-0027-3

Waldis, M., Grob, U., Pauli, C. \& Reusser, K. (2010). Der schweizerische Mathematikunterricht aus der Sicht von Schülerinnen und Schülern und in der Perspektive hochinferenter Beobachterurteile. In K. Reusser, C. Pauli \& M. Waldis (Hrsg.), Unterrichtsgestaltung und Unterrichtsqualität - Ergebnisse einer internationalen und schweizerischen Videostudie zum Mathematikunterricht (S. 171-208). Münster: Waxmann.

Wieser, D. (2008). Literaturunterricht aus Sicht der Lehrenden. Eine qualitative Interviewstudie. Wiesbaden: VS Verlag für Sozialwissenschaften. 
Winkler, I. (2011). Aufgabenpräferenzen für den Literaturunterricht. Eine Erhebung unter Deutschlehrkräften. Wiesbaden: VS Verlag für Sozialwissenschaften.

\section{Förderung}

Die vorliegende Arbeit entstand im Rahmen des Projekts „Die Bedeutung des professionellen Wissens angehender Deutschlehrkräfte für ihre Planung von Unterricht“ (PlanvoLL-D). PlanvoLL-D ist ein Verbundprojekt der Technischen Universität Berlin, der Universität Duisburg-Essen und der Universität zu Köln (Leitung: Prof. Dr. Albert Bremerich-Vos, Dr. Christiane Buchholtz, Prof. Dr. Johannes König), gefördert vom Bundesministerium für Bildung und Forschung (BMBF) im Rahmen der Linie „Kompetenzmodelle und Instrumente der Kompetenzerfassung im Hochschulsektor Validierungen und methodische Innovationen" (KoKoHs). Förderkennzeichen: 01PK15014 A, 01PK15014B, 01PK15014C. Die Daten von Stichprobe 2 (Studierende) wurden im Rahmen des Handlungsfelds „Qualitätssicherung“ des Projekts „Zukunftsstrategie Lehrer*innenbildung Köln - Heterogenität und Inklusion gestalten" (ZuS) der Universität zu Köln erhoben. ZuS wird im Rahmen der gemeinsamen "Qualitätsoffensive Lehrerbildung" von Bund und Ländern aus Mitteln des Bundesministeriums für Bildung und Forschung (BMBF) gefördert.
ORCID

Johannes König

(iD)https://orcid.org/0000-0003-3374-9408

\section{Prof. Dr. Johannes König}

Humanwissenschaftliche Fakultät

Department für Erziehungs- und Sozialwissenschaften Universität zu Köln,

Gronewaldstraße 2

50931 Köln

johannes.koenig@uni-koeln.de

\section{Prof. Dr. Albert Bremerich-Vos}

Fakultät für Geisteswissenschaften Institut für Germanistik

Universität Duisburg-Essen

Berliner Platz 6-8

45127 Essen 